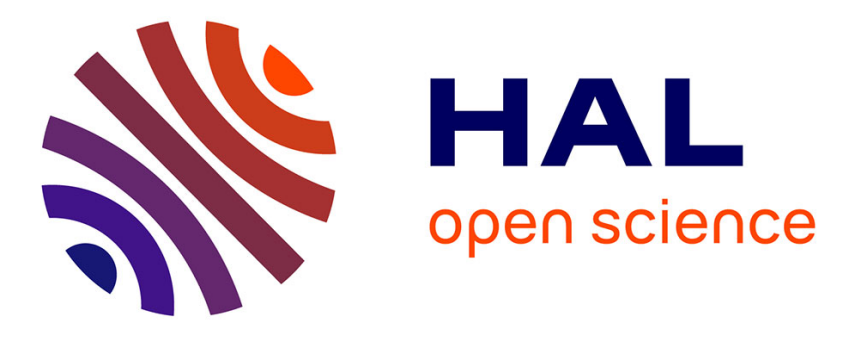

\title{
Bidirectional Nanoparticle Crossing of Oil-Water Interfaces Induced by Different Stimuli: Insight into Phase Transfer
}

Antonio Stocco, Munish Chanana, Ge Su, Peter Cernoch, Bernard P Binks, Dayang Wang

\section{To cite this version:}

Antonio Stocco, Munish Chanana, Ge Su, Peter Cernoch, Bernard P Binks, et al.. Bidirectional Nanoparticle Crossing of Oil-Water Interfaces Induced by Different Stimuli: Insight into Phase Transfer. Angewandte Chemie International Edition, 2012, 51 (38), pp.9647. 10.1002/anie.201203493. hal-00742028

\section{HAL Id: hal-00742028 \\ https://hal.science/hal-00742028}

Submitted on 5 Feb 2013

HAL is a multi-disciplinary open access archive for the deposit and dissemination of scientific research documents, whether they are published or not. The documents may come from teaching and research institutions in France or abroad, or from public or private research centers.
L'archive ouverte pluridisciplinaire HAL, est destinée au dépôt et à la diffusion de documents scientifiques de niveau recherche, publiés ou non, émanant des établissements d'enseignement et de recherche français ou étrangers, des laboratoires publics ou privés. 


\title{
Bidirectional Nanoparticle Crossing of Oil-Water Interfaces Induced by Different Stimuli: Insight into Phase Transfer**
}

\author{
Antonio Stocco, * Munish Chanana, Ge Su, Peter Cernoch, Bernard P. Binks, and Dayang Wang*
}

Gold nanoparticles (NPs) coated with stimuli-responsive polymer brushes are reported that readily transfer from oil to salty water aross the planar interfaces when the environmental temperature is reduced below $5^{\circ} \mathrm{C}$, while they transfer from salty water to oil when the environmental temperature returns to room temperature. The water-to-oil particle transfer after warming is dictated by the ionic strength of the aqueous phase, which is directly associated with the ionic strength reponse of the polymer brushes anchored on the NPs. In contrast, the temperarure onset for the oil-to-water NP transfer after cooling is weakly correlated with the transition temperature of the polymer brushes in either of the bulk phases. The transfer mechanism disparity for the two directions during NP crossing of oil-water interfaces should provide a better thermodynamic picture to describe phase transfer.

The study of NPs at fluid-fluid interfaces is technically important in many traditional industrial processes such as food formulation, crude oil extraction, and mineral flotation. ${ }^{[1]}$ The revealed thermodynamic picture can provide a reasonable model to rationalize and even predict the complicated interfacial phenomena occurring at the molecular level or below. ${ }^{[2,3]}$ Thanks to the experimental and theoretical investigations in the past, one can sensibly elucidate the adsorption of NPs at fluid/fluid interfaces as well as the stability of the emulsions and foams derived from the NP interfacial adsorption. ${ }^{[1,4-7]}$ This success has opened up innovative ways for encapsulation in newly emerging indus-

[*] Dr. A. Stocco, M. Chanana, Dr. P. Cernoch, Prof. D. Wang Max Planck Institute of Colloids and Interfaces 14424 Potsdam (Germany)

Prof. B. P. Binks

Surfactant \& Colloid Group, Department of Chemistry University of Hull, Hull, HU6 7RX (UK)

Dr. A. Stocco

Soft Matter Group, Laboratoire Charles Coulomb

Université Montpellier II et CNRS

34095 Montpellier Cedex 05 (France)

E-mail: antonio.stocco@univ-montp2.fr

G. Su, Prof. D. Wang

Ian Wark Research Institute, University of South Australia

Adelaide, SA 5095 (Australia)

E-mail: dayang.wang@unisa.edu.au

[**] D.W. thanks the Max Planck Society and the Australian Research Council (grant numbers DP1 10104179 and DP120102959) for financial support. A.S. thanks Daniele Cangialosi for discussions. We are grateful to Prof. Helmuth Möhwald for valuable discussion and research support.

9 Supporting information for this article is available on the WWW under http://dx.doi.org/10.1002/anie.201203493. tries such as biocatalysis and interfacial catalysis for biofuel upgrade reactions. ${ }^{[8-10]}$ An important step beyond these investigations is the study of NP crossing of fluid-fluid interfaces. ${ }^{[11-14]}$ This is motivated not only by fundamental interest in using NPs for better mimicking of the interfacial behaviors of ions and molecules but also by increasing technical demand of smart biomedical devices for diagnostics and therapeutic intervention across biological barriers.

Traditional phase transfer of NPs needs surfactants to coat the NPs and thus change the NP surface hydrophobicity. ${ }^{[15]}$ Recent reports have described successful transfer of NPs from aqueous to organic phases across the oil-water interfaces by simply applying an external stimulus such as a change in ionic strength to weaken the hydration of the NP stimuli-responsive polymer coating. ${ }^{[12,13]}$ Similar to the traditional phase transfer, however, this new strategy does not allow reversible transfer of NPs across oil-water interfaces. After removal of the external stimulus, NPs cannot transfer back to the aqueous phase from the organic phase. Additional molecules or polymers were added to help the NPs to build up hydrogen bonding with water even though their polymer coatings are expected to be easily hydrated such as homopolymers of oligo(ethylene glycol) methyl methacrylate) (OEGMA) and poly(ethylene glycol) (PEG). ${ }^{[12,14]}$ This directional interface crossing of NPs is hard to be rationalized as the hydration response of a stimuli-responsive polymer to environmental stimuli is well-known to be reversible in bulk water. On the other hand, this directional interface crossing of NPs largely limits the applicability for labeling or drug delivery across biological barriers as most of the barriers are made up of not one but multiple hydrophobic-hydrophilic interfaces.

To realize the bidirectional interface crossing of NPs and reveal the thermodynamic details governing the phase transfer, herein we have meticulously studied how the environmental temperature and ionic strength affect the phase transfer of Au NPs coated with stimuli-responsive polymer brushes-random copolymers of 2-(2-methoxyethoxy)ethyl methacrylate $\left(\mathrm{MEO}_{2} \mathrm{MA}\right)$ and OEGMA-across oil-water interfaces. As described in our previous reports, ${ }^{[12,13]}$ the polymers were synthesized through atom transfer radical polymerization using disulfide initiators and the polymer used in this work had a $\mathrm{MEO}_{2} \mathrm{MA}$-to-OEGMA molar ratio of 90:10 and is thus denoted as $\mathrm{MEO}_{2} \mathrm{MA}_{90}$-co-OEGMA 10 . Monodiserpse, quasi-spherical, $12 \mathrm{~nm}$ in diameter $\mathrm{Au} \mathrm{NPs}$ were synthesized through citrate reduction of $\mathrm{HAuCl}_{4}$ in water and subsequently coated with $\mathrm{MEO}_{2} \mathrm{MA}_{90}$-co$\mathrm{OEGMA}_{10}$ brushes through ligand exchanges. The resulting $\mathrm{Au} @ \mathrm{MEO}_{2} \mathrm{MA}_{90}$-co-OEGMA 10 NPs were well dispersed in water (Figure $1 \mathrm{~A}$ ) and the hydrodynamic diameters were $20 \mathrm{~nm}$ (see Figure S1 in the Supporting Information). When 

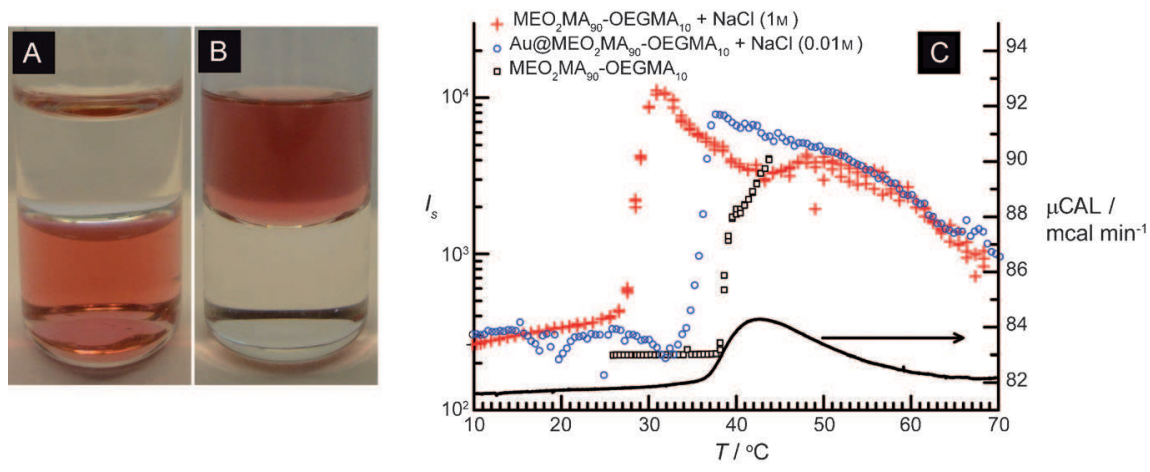

Figure 1. A) Au@MEO $\mathrm{MA}_{90}$-Co-OEGMA 10 NPs well dispersed in water (at room temperature) with a clear toluene phase on top and $B$ ) NP transfer to toluene upon adding salt under stirring. The NP concentration in water is $50 \mathrm{mg} \mathrm{L}^{-1}$. C) Temperature-dependent light scattered intensity profiles of $\mathrm{MEO}_{2} \mathrm{MA}_{90}$-CO-OEGMA $\mathrm{C}_{10}$ copolymer $\left(10 \mathrm{~g} \mathrm{~L}^{-1}\right)$ in pure water (squares) and $1 \mathrm{M} \mathrm{NaCl}$ solution (crosses) and Au@MEO $\mathrm{MA}_{90}$ - $\mathrm{Co}-\mathrm{OEGMA} \mathrm{MPs}_{10} \mathrm{NP}$ with $10 \mathrm{mM} \mathrm{NaCl}$ solution $\left(50 \mathrm{mg} \mathrm{L}^{-1}\right.$, circles). The black curve shows microcalorimetry raw data for the polymer in water. The heating rate $(\delta T / \delta t)$ is $6^{\circ} \mathrm{Ch}^{-1}$.

oil was poured on the top of the aqueous dispersion, a small fraction of $\mathrm{Au} @ \mathrm{MEO}_{2} \mathrm{MA}_{90}$-co-OEGMA 10 NPs adsorbed onto the oil-water interface because of their interfacial activity (see Figure S2 in the Supporting Information). When the oil was a good solvent for $\mathrm{MEO}_{2} \mathrm{MA}_{90}$-co$\mathrm{OEGMA}_{10}$, for example, toluene, NPs readily transferred from water to oil at room temperature when a significant amount of salt, for example, $\mathrm{NaCl}$ was added to the $\mathrm{NP}$ aqueous dispersion (Figure 1B). A complete and quick transfer was observed upon the input of mechanical energy (e.g. stirring, shaking). The NP sizes remained little changed after transfer. All these observations were similar to those reported in our previous reports, ${ }^{[12,13]}$ in which the used $\mathrm{Au}$ NPs were rather polydisperse and produced by using (disulfide-functionalized) $\mathrm{MEO}_{2} \mathrm{MA}_{90}$-co-OEGMA $\mathrm{A}_{10}$ brushes as stabilizers during $\mathrm{NaBH}_{4}$ reduction of $\mathrm{HAuCl}_{4}$. This independence of the production protocol suggests the water-to-oil transfer of $\mathrm{Au} @ \mathrm{MEO}_{2} \mathrm{MA}_{90}$-co-OEGMA ${ }_{10}$ NPs is dominated by the $\mathrm{MEO}_{2} \mathrm{MA}_{90}$-co-OEGMA 10 coating.

The water-to-oil transfer of the NPs was also implemented by increasing the environmental temperature, which, however, occurred only in the presence of salt in the aqueous dispersion. This demonstrates the ionic strength of the aqueous dispersion of NPs plays a dominant role during the water-to-oil NP transfer across the interfaces, highlighting the stimuli-response difference between $\mathrm{MEO}_{2} \mathrm{MA}_{90}$-co$\mathrm{OEGMA}_{10}$ and $\mathrm{Au} @ \mathrm{MEO}_{2} \mathrm{MA}_{90}$-co-OEGMA 10 NPs. Figure $1 \mathrm{C}$ shows that the scattered intensities of the aqueous solutions of $\mathrm{MEO}_{2} \mathrm{MA}_{90}$-co-OEGMA 10 copolymer increase abruptly above $40^{\circ} \mathrm{C}$, suggesting the polymers become hydrophobic and aggregate in water. This first-order-like hydrophilic-to-hydrophobic transition is also verified by microcalorimetry ( $\mu \mathrm{CAL})$ measurements and the transition temperature-lower critical solution temperature (LCST)shows a strong dependence on the salt concentration; it shifts from 40 to $28^{\circ} \mathrm{C}$ when a significant amount of salt (1M) is added to the aqueous polymer solution. In contrast, $\mathrm{Au} @ \mathrm{MEO}_{2} \mathrm{MA}_{90}$-co-OEGMA 10 NPs exhibit a LCST only in the presence of salt in their aqueous dispersion; the LCST is around $35^{\circ} \mathrm{C}$ at an ionic strength of $10 \mathrm{~mm}$. Temperature alone cannot cause the hydrophilic-to-hydrophobic transition on the NP surfaces. This highlights that the surface hydrophobicity of $\mathrm{Au} @ \mathrm{MEO}_{2} \mathrm{MA}_{90}$-coOEGMA $_{10}$ NPs is more sensitive to the ionic strength rather than the temperature of the surrounding media. The stimuli-response difference between free $\mathrm{MEO}_{2} \mathrm{MA}_{90}$-coOEGMA $_{10}$ brushes and those coating $\mathrm{Au}$ NPs should be due to the fact that the polymer brushes are anchored on the NPs and thus not able to alter their conformations so freely as they can in solution. This also suggests that the surface hydrophilic-to-hydrophobic transition of the NPs should be mainly governed by the hydrogenbonding not of the entire polymer brush shells but their outmost layers with water; otherwise the NP should show a noticeable temperature-response in the absence of salt.

After $\mathrm{Au} @ \mathrm{MEO}_{2} \mathrm{MA}_{90}$-co-OEGMA 10 NPs transferred into oil, the use of pure water to replace salty water could not induce the NP transfer back to water at room temperature (about $10^{\circ} \mathrm{C}$ lower than the NP LCST of $35^{\circ} \mathrm{C}$ at the ionic strength of $10 \mathrm{~mm}$ ). This underlines the ionic strength-induced water-to-oil transfer of the NPs is irreversible, which is in contradiction to the reversible responses of both $\mathrm{Au} @ \mathrm{MEO}_{2} \mathrm{MA}_{90}$-co-OEGMA 10 NPs and $\mathrm{MEO}_{2} \mathrm{MA}_{90}$-co$\mathrm{OEGMA}_{10}$ to both ionic strength and temperature in (salty) water (Figure 1C). Surprisingly, the transfer of $\mathrm{Au} @ \mathrm{MEO}_{2} \mathrm{MA}_{90}$-co-OEGMA 10 NPs from oil back to water was observed only when the environmental temperature was reduced below $5^{\circ} \mathrm{C}$ (Figure $2 \mathrm{~A}$ ). At that low temperature, the

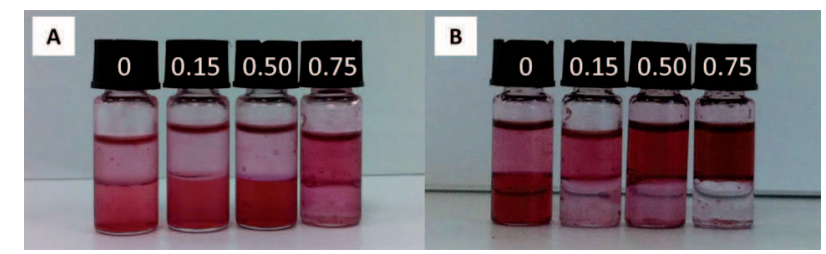

Figure 2. A) Transfer of $A u @ M E O_{2} M_{90}-\mathrm{Co}-O E G M A_{10} N P s$ from toluene to the aqueous phase at $2^{\circ} \mathrm{C}$ after shaking. B) NP transfer from the aqueous phase $(10 \mathrm{~mm} \mathrm{NaCl})$ to toluene upon warming to room temperature after shaking. The numbers above the glass vials are the ionic strengths in the aqueous phase. The NP concentration in water is $50 \mathrm{mg} \mathrm{L}^{-1}$.

NPs could transfer back not only to pure water but also to salty water with an ionic strength as high as $0.50 \mathrm{M}$; no NP transfer was visible at the ionic strength $\geq 0.75 \mathrm{M}$ in water. The input of mechanical energy (e.g. stirring and shaking) facilitated a complete and quick transfer. When the environmental temperature is returned to room temperature, the NP transfer back to oil from salty water is observed (Figure 2B). This underlines a bidirectional interface crossing of the NPs. 
Since no NP transfer from water to toluene has been observed in the absence of salt (Figure 2B), this apparent temperatureinduced water-to-oil NP transfer is still driven by the ionic strength. This suggests that the observed bidirectional interface crossing of the NPs is actually not a reversible process in terms of stimuli-response.

The phase transfer of $\mathrm{Au} @ \mathrm{MEO}_{2} \mathrm{MA}_{90}$-co-OEGMA ${ }_{10}$ NPs across the oil-water interface is a two-step process: NP attachment from a liquid phase onto the interface (desolvation) and detachment from the interface to the other liquid phase (re-solvation). Figure 3 shows that the light

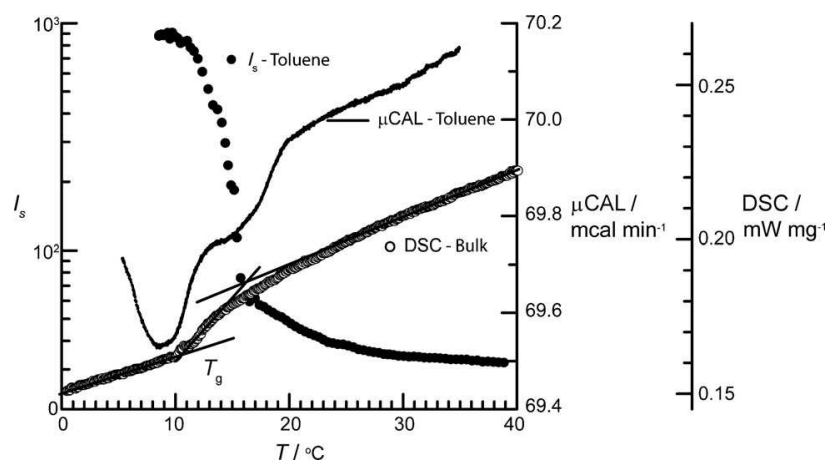

Figure 3. Temperature-dependent light scattered intensity of dispersions of $\mathrm{Au} @ \mathrm{MEO}_{2} \mathrm{MA}_{90}$-Co-OEGMA 10 NPs in toluene $\left(50 \mathrm{mg} \mathrm{L}^{-1}, \delta T /\right.$ $\delta t=6^{\circ} \mathrm{Ch}^{-1}$ ) (solid circles). Microcalorimetry raw data of $\mathrm{MEO}_{2} \mathrm{MA}_{90^{-}}$ co-OEGMA 10 copolymer in toluene $\left(10 \mathrm{~g} \mathrm{~L}^{-1}, \delta T / \delta t=6^{\circ} \mathrm{Ch}^{-1}\right)$ (blue curve). Differential scanning calorimetry (DSC) raw data of dry $\mathrm{MEO}_{2} \mathrm{MA}_{90}$-Co-OEGMA $10\left(\delta T / \delta t=600^{\circ} \mathrm{Ch}^{-1}\right)$ (red open circles).

scattered intensities of the dispersions of $\mathrm{Au} @ \mathrm{MEO}_{2} \mathrm{MA}_{90^{-}}$ co-OEGMA ${ }_{10}$ NPs in toluene show an abrupt increase below $15^{\circ} \mathrm{C}$ during cooling. In a similar temperature range, the calorimetry data indicate a $\mu \mathrm{CAL}$ ramp of $\mathrm{MEO}_{2} \mathrm{MA}_{90}$-co$\mathrm{OEGMA}_{10}$ in toluene, which corresponds to a glass transition with a heat capacity $\left(C_{\mathrm{P}}\right)$ change of $0.6-0.9 \mathrm{Jg} \mathrm{g}^{-1}$, determined by differential scanning calorimetry (DSC). This suggests that the $\mathrm{MEO}_{2} \mathrm{MA}_{90}$-co-OEGMA ${ }_{10}$ becomes glassy below $10^{\circ} \mathrm{C}$ and less soluble in toluene. ${ }^{[16,17]}$ In this scenario, significant adsorption of Au@ $\mathrm{MEO}_{2} \mathrm{MA}_{90}$-co-OEGMA 10 NPs from toluene to the toluene-water interface is expected when the environmental temperature is reduced below $10^{\circ} \mathrm{C}$. Furthermore, NP crossing of the interface to disperse into water should also be expected as (pure) water is an excellent solvent for both $\mathrm{MEO}_{2} \mathrm{MA}_{90}$-co-OEGMA 10 and their coated NPs at room temperature. However, our experimental observations suggest that further temperature reduction is needed for $\mathrm{Au} @ \mathrm{MEO}_{2} \mathrm{MA}_{90}$-co-OEGMA 10 NPs to realize the oil-towater transfer. The temperature $\left(<5^{\circ} \mathrm{C}\right)$ required for the oilto-water NP transfer is far lower than the LCST of the NPs $\left(35^{\circ} \mathrm{C}\right.$ at an ionic strength of $10 \mathrm{~mm}$, Figure $\left.1 \mathrm{C}\right)$ and the glass transition temperature of the NPs $\left(15^{\circ} \mathrm{C}\right.$ in toluene, Figure 3$)$. This suggests that the re-hydration of $\mathrm{Au} @ \mathrm{MEO}_{2} \mathrm{MA}_{90}-\mathrm{co}-$ $\mathrm{OEGMA}_{10}$ NPs originally dispersed in oil at the oil-water interface is rather difficult compared with that of the NPs dispersed in water.
To explain the aforementioned experimental observations, we developed a theoretical framework to describe $\mathrm{Au} @ \mathrm{MEO}_{2} \mathrm{MA}_{90}$-co-OEGMA 10 NP crossing of oil-water interfaces in two directions (see the Supporting Information for computational details). The free-energy difference of NPs in oil and water should be the driving force for the NPs to cross the interface. For an isolated NP, the energy as a function of its position $(z)$ relative to a simple interface can be described in terms of the wetting energy $E_{\mathrm{W}}(z)$ given in Equation (1): ${ }^{[3]}$

$E_{\mathrm{W}}(z)=\sigma_{1} A_{1}(z)+\sigma_{2} A_{2}(z)-\gamma A_{0}(z)$

where $\sigma_{1}$ and $\sigma_{2}$ are the surface energies of the NP in oil and water $\left(\sigma_{1}=1 \mathrm{mN} \mathrm{m}^{-1}\right.$ and $\left.\sigma_{2}=1.6 \mathrm{mN} \mathrm{m}^{-1}\right), A_{i}$ are the areas occupied by the NP in each medium and $\gamma A_{0}(z)$ is the energy gain corresponding to the removal of the interface (Experimental $\gamma$ is shown in Figure S2 in Supporting Information). The transfer of a NP from water to oil takes places when $E_{1}$ (free-energy plateau value in oil) is smaller than $E_{2}$ (freeenergy plateau value in water), namely when $\sigma_{1}<\sigma_{2}$ in Figure 4. This can be realized by adding salt into water

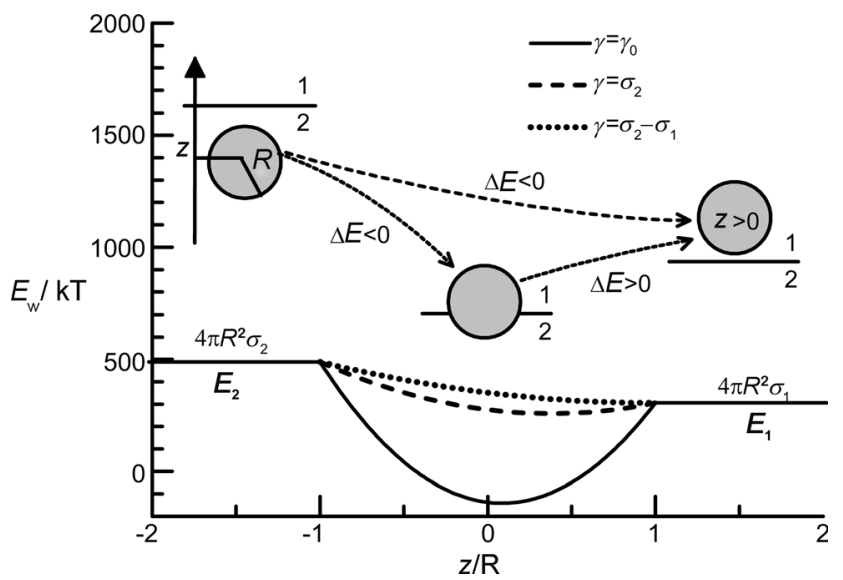

Figure 4. Free-energy of a NP $(R=10 \mathrm{~nm})$ versus its position $(z)$ relative to the interface between medium 1 (toluene) and medium 2 (water) evaluated from Equation (1) for $\gamma=\gamma_{0}\left(7 \mathrm{mN} \mathrm{m}^{-1}\right), \sigma_{2}$, and $\sigma_{2}-\sigma_{1}$.

according to our previous study of the surface energies of $\mathrm{MEO}_{2} \mathrm{MA}_{90}$-co-OEGMA 10 anchored to planar gold surfaces. ${ }^{[12,13]}$ From Equation (1), the adsorption of NPs of $20 \mathrm{~nm}$ in diameter to oil-salty water interfaces is thermodynamically favorable; the adsorption energy being about $600 \mathrm{kT}$. Our modeling, however, also indicates a pronounced potential well of about $10^{2} k T$ in the interfacial region. ${ }^{[3]}$ This can explain the additional energy input such as stirring or shaking that is needed for NP crossing of interfaces (Figure 4). When the NPs are already adsorbed at the interfaces, moreover, the real interfacial tension should possibly be as low as $\gamma=\sigma_{2}-\sigma_{1}$, with the latter condition implying vanishing of the potential well at the interface.

In a realistic description, not only wetting energy but also other interactions should be taken into account, including van der Waals attractions $\left(E_{\mathrm{A}}\right)$, electrostatic repulsion $\left(E_{\mathrm{R}}\right)$, 
hydrophobic attraction $\left(E_{\mathrm{HB}}\right)$, osmotic repulsion $\left(E_{\mathrm{O}}\right)$, and the NP shell (polymer) interaction with bulk media $\left(E_{\mathrm{P}}\right)$. Accordingly, the free-energy of a NP at an interface is expressed in Equation (2),

$E(z)=E_{\mathrm{W}}+E_{\mathrm{A}}+E_{\mathrm{R}}+E_{\mathrm{HB}}+E_{\mathrm{O}}+E_{\mathrm{P}}$

where $E_{\mathrm{A}}$ and $E_{\mathrm{R}}$ were described by the DLVO theory. ${ }^{[2]} E_{\mathrm{HB}}$ was calculated using literature values for $\mathrm{Au} \mathrm{NPs} .^{[18]}$ The approximation for repulsive interaction between polymercovered surfaces was used to calculate $E_{\mathrm{O}}{ }^{\left[{ }^{[2]}\right.} E_{\mathrm{p}}$ can be evaluated from $E_{\mathrm{p}}=n_{1} f_{1}\left(\chi_{\mathrm{FH}, 1}\right)+n_{2} f_{2}\left(\chi_{\mathrm{FH}, 2}\right)$, where $n_{i}$ are the number of polymer chains, and $f_{i}$ is the free-energy associated with the Flory-Huggins parameters $\left(\chi_{\mathrm{FH}, i}\right)$ of the polymer shells in each medium. ${ }^{[4,16]} \mathrm{MEO}_{2} \mathrm{MA}_{90}$-co-OEGMA ${ }_{10}$ can be simplified as a mixture of PEG and poly(methyl methacrylate) (PMMA) units. Toluene is a good solvent for both PEG and PMMA units $\left(\chi_{\mathrm{FH}, 1}(\mathrm{PEG})<1 / 2\right.$ and $\left.\chi_{\mathrm{FH}, 1}(\mathrm{PMMA})<1 / 2\right)$, namely, $\chi_{\mathrm{FH}, 1}<1 / 2 \cdot{ }^{[19-21]}$ In contrast, water is a good solvent for PEG units $\left(\chi_{\mathrm{FH}, 2}(\mathrm{PEG}) \approx 1 / 2\right)$ but poor for PMMA units $\left(\chi_{\mathrm{FH}, 2}(\mathrm{PMMA})>1 / 2\right) \cdot{ }^{[19-21]}$ Although Au@MEO $\mathrm{MA}_{90}$-coOEGMA $_{10}$ NPs disperse well in water, we still expect $\chi_{\mathrm{FH}, 2} \approx 1 / 2$ as the NPs show a noticeable surface activity in water (see Figure S2 in the Supporting Information). The hydrophilic-to-hydrophobic transition of the polymer shells of $\mathrm{Au} @ \mathrm{MEO}_{2} \mathrm{MA}_{90}$-co-OEGMA 10 NPs, shown in Figure 1C, suggests $\chi_{\mathrm{FH}, 2}<1 / 2$ upon adding salt to water. As such, toluene represents a favorable energetic state for the NP shells of $\mathrm{MEO}_{2} \mathrm{MA}_{90}$-co-OEGMA ${ }_{10}$, but the NP transfer from pure and especially salty water does not occur at room temperature even with the help of stirring or shaking to overcome the deep potential well at the interface. Furthermore, as shown in Figure $5 \mathrm{~A}$, the interplay between $E_{\mathrm{A}}, E_{\mathrm{R}}, E_{\mathrm{HB}}, E_{\mathrm{O}}$, and $E_{\mathrm{P}}$ results in an energy barrier (about $10^{2} k T$ ) in water close to the interface to prevent the interfacial adsorption of NPs, which rapidly vanishes upon adding a reasonable amount of salt $(>1 \mathrm{~mm})$ to water. Note that adding salt corresponds to an increase of number density of ions in solution and a decrease in the Debye screening length which lead to a decrease of $E_{\mathrm{R}}$. This accordingly underlines the dominant role of the ionic strength during the water-to-oil transfer of $\mathrm{Au} @ \mathrm{MEO}_{2} \mathrm{MA}_{90}$-co-OEGMA 10 NPs.

According to our modeling, the transfer of a NP from oil to water needs $E_{1}>E_{2}$. Since the NP is now in oil, only $\chi_{\mathrm{FH}, 1}$ among various parameters included in Equation (2) is noticeably sensitive to the environmental variables. Figure $5 \mathrm{~B}$ shows the dramatic effect of the change in the magnitude of $\chi_{\mathrm{FH}, 1}$ and $\chi_{\mathrm{FH}, 2}$ of the $\mathrm{Au} @ \mathrm{MEO}_{2} \mathrm{MA}_{90}$-CO-OEGMA 10 NPs on $E_{1}$ and $E_{2}$. As shown in Figure 3, the polymer brush shells of $\mathrm{Au} @ \mathrm{MEO}_{2} \mathrm{MA}_{90}$-co-OEGMA ${ }_{10}$ NPs incur a glass transition below $15^{\circ} \mathrm{C}$ in toluene, thus suggesting that toluene is not a good solvent for the $\mathrm{MEO}_{2} \mathrm{MA}_{90}$-co-OEGMA 10 coating. On the other hand, we also found that water/toluene (1:1) emulsions stabilized by $\mathrm{Au} @ \mathrm{MEO}_{2} \mathrm{MA}_{90}$-co-OEGMA 10 NPs were always of oil-in-water-type at room temperature regardless of the phase where NPs were originally dispersed (see Figure S3 in the Supporting Information). This suggests that the hydration of the PEG units renders Au@MEO $\mathrm{MAA}_{90}$-coOEGMA $_{10}$ NPs in toluene interfacially active. Taking into

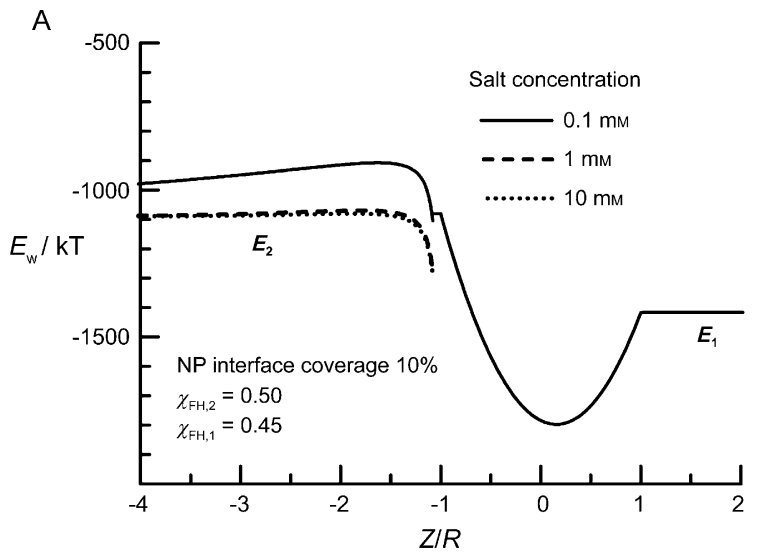

B

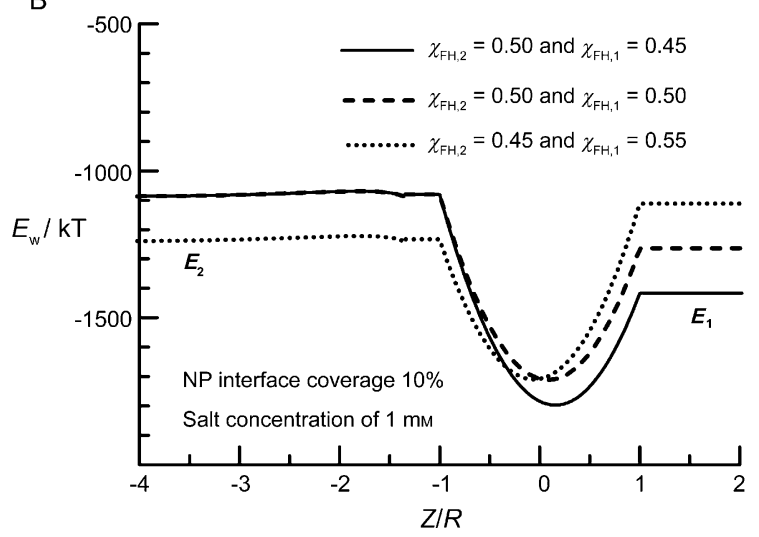

Figure 5. Free-energy of a NP (with a radius $R$ of $10 \mathrm{~nm}$ ) versus its position $z$ relative to the toluene-water interface with $10 \%$ NP coverage calculated from Equation (2). The energy profile dependence on A) the ionic strength and B) the interaction parameters in water $\left(\chi_{\mathrm{FH}, 2}\right)$ and in toluene $\left(\chi_{\mathrm{FH}, 1}\right)$ are given.

account the aforementioned surface activity of the NPs in water, therefore, we firstly set both $\chi_{\mathrm{FH}, 1}$ and $\chi_{\mathrm{FH}, 2}$ equal to $1 / 2$. The value of $E_{1}$ calculated from Equation (2) noticeably increases but is not larger than that of $E_{2}$. According to our modeling, only when we set $\chi_{\mathrm{FH}, 1}=0.55$ and $\chi_{\mathrm{FH}, 2}=0.45$, can we make $E_{1}$ significantly larger than $E_{2}$ and thus water as the thermodynamically favorable phase for NPs to transfer from oil; the change in the total energy $\Delta E$ is about $300 \mathrm{kT}$ comparable to that observed during the water-to-oil transfer shown in Figures 4 and $5 \mathrm{~A}$. The Flory-Huggins parameter $\chi_{\mathrm{FH}, 1}$ of 0.55 can be rationalized especially taking into account the glass transition hysteresis of the polymer shells of $\mathrm{Au} @ \mathrm{MEO}_{2} \mathrm{MA}_{90}$-co-OEGMA 10 NPs and the complete vitrification needs further cooling far below $10^{\circ} \mathrm{C}$. The FloryHuggins parameter $\chi_{\mathrm{FH}, 2}$ of 0.45 suggests excellent hydration of the $\mathrm{MEO}_{2} \mathrm{MA}_{90}-\mathrm{co}-\mathrm{OEGMA}{ }_{10}$ shells in water. It has been reported that although PEG can be rapidly hydrated through one ethylene oxide unit associated with one water molecule, further cooling is needed for equilibrium hydration where one ethylene oxide unit is associated with three water molecules to reach a helical polymer conformation. ${ }^{[22]}$ Furthermore, water molecules become highly associated around $4{ }^{\circ} \mathrm{C}$ and tend to adsorb onto hydrophobic surfaces or dissolve hydrophobic species. $^{[23]}$ In this scenario, hydration of the PMMA 
units of the $\mathrm{MEO}_{2} \mathrm{MA}_{90}$-co-OEGMA 10 shells might be expected. As such, a Flory-Huggins parameter $\chi_{\mathrm{FH}, 2}$ of 0.45 should be feasible only at fairly low temperatures (below $5^{\circ} \mathrm{C}$ ). Hydration is expected to be ionic strength dependent. As suggested in Figure $2 \mathrm{~A}$, however, this dependence is significantly suppressed below $5^{\circ} \mathrm{C}$; NPs can transfer from oil back to a $0.50 \mathrm{M} \mathrm{NaCl}$ solution. The value of $\chi_{\mathrm{FH}, 1}$ could be further increased by adding hexane, the precipitating solvent of $\mathrm{MEO}_{2} \mathrm{MA}_{90}$-co-OEGMA 10 , to toluene, which could transfer $\mathrm{Au} @ \mathrm{MEO}_{2} \mathrm{MA}_{90}$-co-OEGMA 10 NPs back only to pure water at room temperature (see Figure $\mathrm{S} 4$ in the Supporting Information). This also underlines the cooling below $5^{\circ} \mathrm{C}$ can improve the hydration of $\mathrm{Au} @ \mathrm{MEO}_{2} \mathrm{MA}_{90}$-co-OEGMA 10 NPs in salty water. Note that the deep potential well of about $10^{2} k T$ in the interfacial region, shown in Figures 4 and 5 , is expected to significantly increase with the nanoparticle size. We observed that nanoparticles above $30 \mathrm{~nm}$ could not cross oil/water interface but attached at the interfaces, similar to our previsous study; ${ }^{[13]}$ cooling below $5^{\circ} \mathrm{C}$ could not detach the nanoparticles from the interfaces (see Figure S6 in the Supporting Information).

In conclusion, we have successfully demonstrated bidirectional phase transfer of NPs across oil-water interfaces by deliberately engineering their surface energy with environmental stimuli. The water-to-oil NP transfer is governed by the ionic strength in water, which is directly associated with the stimuli-response of the NP surfaces. But the oil-to-water transfer occurs at temperatures below $5^{\circ} \mathrm{C}$, which is weakly associated with the stimuli-response of the NP surfaces. The present stimuli-responsive NPs should provide innovative probes to study the thermodynamic details at interfaces and smart carriers for delivery across interfaces. We have developed a simple framework to catch most of the features of NPs crossing interfaces, which should also give new insights into interface crossing of ions and molecules. We hope also to stimulate some theoretical work to treat NP phase transfer in more detail by including, for example line tension, surface water structures, and complicated modeling of NP partitioning at interfaces. ${ }^{[5,6,24-26]}$

Received: May 7, 2012

Revised: July 12, 2012

Published online:
[1] B. Binks, Curr. Opin. Colloid Interface Sci. 2002, 7, 21-41.

[2] J. N. Israelachvili, Intermolecular and Surface Forces, Academic Press, New York, 2nd ed, 1991.

[3] P. Pieranski, Phys. Rev. Lett. 1980, 45, 569-572.

[4] M. E. Leunissen, A. van Blaaderen, A. Hollingsworth, M. T. Sullivan, P. Chaikin, Proc. Natl. Acad. Sci. USA 2007, 104, $2585-$ 2590.

[5] S. Kutuzov, J. He, R. Tangirala, T. Emrick, T. Russell, A. Böker, Phys. Chem. Chem. Phys. 2007, 9, 6351-6358.

[6] L. Isa, E. Amstad, K. Schwenke, E. Del Gado, P. Ilg, M. Kroger, E. Reimhult, Soft Matter 2011, 7, 7663-7675.

[7] A. Stocco, E. Rio, B. Binks, D. Langevin, Soft Matter 2011, 7, $1260-1267$.

[8] S. Crossley, J. Faria, M. Shen, D. E. Resasco, Science 2010, 327, $68-72$.

[9] M. Li, D. C. Green, J. L. Ross, J. L. R. Anderson, B. P. Binks, S. Mann, Chem. Sci. 2011, 2, 1739-1745.

[10] C. Wu, S. Bai, M. Ansorge-Schumacher, D. Wang, Adv. Mater. 2011, 23, 5694-5699.

[11] Z. Bai, T. Lodge, Langmuir 2010, 26, 8887-8892.

[12] E. W. Edwards, M. Chanana, D. Wang, H. Möhwald, Angew. Chem. 2008, 120, 326-329; Angew. Chem. Int. Ed. 2008, 47, 320 323.

[13] E. W. Edwards, M. Chanana, D. Wang, J. Phys. Chem. C 2008, $112,15207-15219$.

[14] Z. Mao, J. Guo, S. Bai, T.-L. Nguyen, H. Xia, Y. Huang, P. Mulvaney, D. Wang, Angew. Chem. 2009, 121, 5053-5056; Angew. Chem. Int. Ed. 2009, 48, 4953-4956.

[15] M. Sastry, Curr. Sci. 2003, 85, 1735-1745.

[16] U. Gedde, Polymer Physics, Kluwer Academic Publishers, Dordrecht, 1995.

[17] Z. Hu, T. Cai, C. Chi, M. Marquez, US Application Publication, US 2010/0076105A1, 2010.

[18] L. Xu, G. Han, J. Hu, Y. He, J. Pan, Y. Li, J. Xiang, Phys. Chem. Chem. Phys. 2009, 11, 6490-6497.

[19] Y. Bae, J. Shim, D. Soane, J. Prausnitz, J. Appl. Polym. Sci. 1993, 47, $1193-1206$.

[20] A. Eliassi, H. Modarress, G. Mansoori, J. Chem. Eng. Data 1999, $44,52-55$.

[21] C. Özdemir, A. Guner, J. Appl. Polym. Sci. 2006, 101, 203-216.

[22] N. B. Graham, M. Zulfiqar, N. E. Nwachuku, A. Rashid, Polymer 1989, 30, 528-533.

[23] J. L. Finney, Phil. Trans. R. Soc. Lond. B 2004, 359, 1145-1165.

[24] A. Almusallam, Phys. Chem. Chem. Phys. 2010, 12, $12198-$ 12207.

[25] V. Garbin, J. Crocker, K. Stebe, Langmuir 2012, 28, 1633-1667.

[26] H. Wi, S. Cingarapu, K. Klabunde, B. Law, Langmuir 2011, 27, 9979-9984.

Keywords: interfaces - nanoparticles - phase transfer .

soft matter 


\section{Angewandte}

Communications

\section{Communications}

Soft Matter

A. Stocco,* M. Chanana, G. Su,

P. Cernoch, B. P. Binks,

D. Wang*

\section{IIII-IIII}

Bidirectional Nanoparticle Crossing of Oil-Water Interfaces Induced by Different Stimuli: Insight into Phase Transfer

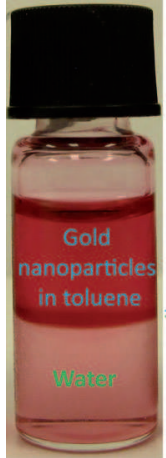

lonic strength increase in water

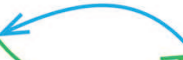

Environmental

temperature

below $5^{\circ} \mathrm{C}$
Swap transactions: Bidirectional spontaneous transfer of gold nanoparticles coated with stimuli-responsive polymer brushes across oil-water interfaces has been implemented (see picture). The water-to-oil transfer of the gold nanoparticles is dictated by the ionic strength in water, while the nanoparticle oil-towater transfer occurs only when the environmental temperature is reduced below $5^{\circ} \mathrm{C}$ 